\title{
The shell model of electron structure of negative hydrogen ion
}

\author{
Vavrukh M., Dzikovskyi D., Stelmakh O. \\ Ivan Franko National University of Lviv, \\ 8 Kyrylo and Methodiy Str., 79005, Lviv, Ukraine
}

(Received 29 January 2019; Revised 5 June 2019; Accepted 6 June 2019)

\begin{abstract}
In the frame of non-relativistic approximation, a compact approximate solution of the Schrödinger equation for the ion of $H^{-}$has been obtained in the form of product for Legendre polynomials and variational functions of the Schull-Löwdin type. The accuracy of calculation of ion energy is of the same order that the results obtained using the multiparametric functions of Hylleraas and Pekeris.
\end{abstract}

Keywords: negative hydrogen ion, reference system approach, variation method, energy of ionization.

2000 MSC: $70 \mathrm{~F} 05$

UDC: $523.9,523.9-7,523.9-47$

DOI: $10.23939 / \mathrm{mmc} 2019.01 .144$

\section{Introduction}

The negative hydrogen ion is an example of the simplest quantum-mechanical object with the strong inter-electron correlations, which determines the independence and importance of the problem which we consider. The relevance of this problem for astrophysics is due to the fact that the negative hydrogen ions are one of the main factors of the formation of continuous absorption coefficient in the photospheres of Solar-like stars [1-5]. For the calculations of spectral dependence of the photoionization cross-section for $H^{-}$ions it is useful to have a compact wave function which differs from the cumbersome variational functions of Hylleraas [6] or function of Pekeris [7], which have tens or hundreds of terms. Such functions are based on the principle - the more variational parameters they have, the better they are. But the construction of such functions does not take into account the specificity of inter-electron correlations in atomic systems what causes a self-consistent distribution of electron density. Unreasonable complexity of such wave functions make it difficult to analyze the role of inter-electron correlations in the twoelectron systems, as well as their use in applied problems, in particular in astrophysics, solid state physics and in nanostructural systems.

The description of the quantum state of the $H^{-}$ion in the non-relativistic approximation is reduced to finding solution of the Schrödinger equation for the motion of two electrons in the field of static proton

$$
\left\{\hat{h}\left(\mathbf{r}_{1}\right)+\hat{h}\left(\mathbf{r}_{2}\right)+\hat{v}\left(\mathbf{r}_{1}, \mathbf{r}_{2}\right)\right\} \Psi_{-}\left(\mathbf{r}_{1}, \mathbf{r}_{2}\right)=E_{-} \Psi_{-}\left(\mathbf{r}_{1}, \mathbf{r}_{2}\right),
$$

where

$$
\hat{h}(\mathbf{r})=-\frac{\hbar^{2} \nabla^{2}}{2 m}-\frac{e^{2}}{r}, \quad \hat{v}\left(\mathbf{r}_{1}, \mathbf{r}_{2}\right)=e^{2}\left|\mathbf{r}_{1}-\mathbf{r}_{2}\right|^{-1} .
$$

There are known only approximate solutions for this problem. Because of strong inter-electron correlations the Hartree-Fock approximation is not applicable here (in the self-consistent approach $E_{-}>-0.945 \ldots$ Ry) [8]. The simplest variational function, which approximately take into account only radial correlations of electrons is the function of Schull-Löwdin [9]

$$
\Psi\left(\boldsymbol{\rho}_{1}, \boldsymbol{\rho}_{2}\right)=N_{a, b}\left\{\exp \left[-a \rho_{1}-b \rho_{2}\right]+\exp \left[-b \rho_{1}-a \rho_{2}\right]\right\}, \quad N_{a, b}=\left\{1+(4 a b)^{3}(a+b)^{-6}\right\}^{-1 / 2},
$$


where $\boldsymbol{\rho}_{i} \equiv \mathbf{r}_{i} a_{0}^{-1}, a_{0}$ is the Bohr radius, $a$ and $b$ are the variational parameters. At $a=1.0392$, $b=0.2832$ the function (3) yields the energy $E_{-}=-1.0266 \ldots$ Ry. The function (3) is the symmetrical combination of two $1 s$-type orbits. The maximum probability of the one-particle density distribution for first of them corresponds to $\rho \simeq a^{-1}$, and for the second one $-\rho \simeq b^{-1}$, which provides within this approximation a minimum of matrix element for the potential energy of inter-electron repulsion. The function (3) corresponds to the dual-shell model of the electron density distribution in the $\mathrm{H}^{-}$ion.

The three-parametric variational Chandrasekar's function [2]

$$
\Psi\left(\boldsymbol{\rho}_{1}, \boldsymbol{\rho}_{2}\right)=M\left\{\exp \left[-a \rho_{1}-b \rho_{2}\right]+\exp \left[-b \rho_{1}-a \rho_{2}\right]\right\}\left\{1+c\left|\boldsymbol{\rho}_{1}-\boldsymbol{\rho}_{2}\right|\right\}
$$

generalizes the expression (3) and gives the energy $E_{-}=-1.05184 \ldots$ Ry at $\alpha=1.077758, \beta=$ $0.47758, c=0.31214$ approximately accounting also for the angular correlations in the electron charge distribution. As follows from the calculations based on simple wave functions with the small number of variational parameters, the contributions of radial and angular correlations to the ionization energy are almost of the same order $(\approx 0.0275 \ldots$ Ry $)$.

In the works of Hylleraas [6], Kinoshita [10], Hart and Herzberg [11], Schwartz (see [3]) and Pekeris [7] the variational calculations of wave functions and energies of ground state of the $H^{-}$ion, the helium atom and helium-like positive ions were done with high accuracy by using trial functions with large number of variation parameters. In particular, Hart and Herzberg [11] were calculated the wave function and energy of ground state of the helium and helium-like ions based on 20-parametric Hylleraas function

$$
\begin{aligned}
\Psi\left(\mathbf{r}_{1}, \mathbf{r}_{2}\right)= & N \exp \left\{-\frac{1}{2} k s\right\}\left\{1+\chi_{1} u+\chi_{2} t^{2}+\chi_{3} s+\chi_{4} s^{2}+\chi_{5} u^{2}\right. \\
& +\chi_{6} s u+\chi_{7} t^{2} u+\chi_{8} u^{3}+\chi_{9} t^{2} u^{2}+\chi_{10} s t^{2}+\chi_{11} s^{3}+\chi_{12} t^{2} u^{4}+\chi_{13} u^{4} \\
& \left.+\chi_{14} u^{5}+\chi_{15} t^{2} u^{3}+\chi_{16} s^{2} t^{2}+\chi_{17} s^{4}+\chi_{18} s u t^{2}+\chi_{19} t^{4}\right\} \\
s= & \rho_{1}+\rho_{2}, \quad t=\rho_{1}-\rho_{2}, \quad u=\left|\boldsymbol{\rho}_{1}-\boldsymbol{\rho}_{2}\right|
\end{aligned}
$$

in which $k, \chi_{1}, \ldots, \chi_{19}$ are the variational parameters and $\boldsymbol{\rho}_{1}, \boldsymbol{\rho}_{2}$ and their absolute values are expressed in units $a_{0}$. This function leads to the value of energy $E_{-}=-1.055289 \ldots$ Ry. The Schwartz's 70parametric function has the same structure and gives the energy $E_{-}=-1.055495 \ldots$ Ry [3]:

$$
\Psi\left(\mathbf{r}_{1}, \mathbf{r}_{2}\right)=\exp \left\{-\frac{1}{2} k s\right\} \sum_{1}^{70} \chi_{l m n} \rho_{12}^{l}\left(\rho_{1}^{m} \rho_{2}^{n}+\rho_{1}^{n} \rho_{2}^{m}\right) .
$$

In his work Pekeris [7] used the parametric coordinates

$$
u=\varepsilon\left(\rho_{2}-\rho_{1}-\rho_{12}\right), \quad v=\varepsilon\left(\rho_{1}-\rho_{2}+\rho_{12}\right), \quad w=2 \varepsilon\left(\rho_{1}+\rho_{2}-\rho_{12}\right),
$$

which are independent and vary within the limits $(0 \div \infty)$ and $\varepsilon=\{-2 m E\}^{1 / 2} a_{0} \hbar^{-1}$. The wave function is expressed in the form

$$
\Psi\left(\mathbf{r}_{1}, \mathbf{r}_{2}\right)=\exp \{-[u+v+w]\} \sum_{l, m, n \geqslant 0} A(l, n, m) L_{l}(v) L_{m}(w) L_{m}(u),
$$

where $L_{s}(x)$ is the normalized Laguerre polynomials, $A(l, n, m)$ are unknown parameters. According to its structure the function (8) belongs to Hylleraas type functions. During the numerical calculations 444 terms of series were taken into account with $l+m+n \leqslant 16$, although the spectroscopic accuracy (when the error has the order $10^{-6} \mathrm{Ry}$ ) is achieved already with 100 terms of series (8). The ion energy is in the limits

$$
-1.055502028 \ldots \text { Ry }<E_{-}<-1.055502000 \ldots \text { Ry } .
$$

Mathematical Modeling and Computing, Vol.6, No. 1, pp. 144-151 (2019) 
Although the expression (8) is the series of orthogonal functions, however the Laguerre polynomials determine the wave functions of electron in the neutral hydrogen atom and do not take into account the electron correlations. Therefore it is necessary to take into account the huge number series terms.

In the work [12] is used the variational function

$$
\Psi\left(\mathbf{r}_{1}, \mathbf{r}_{2}\right)=\exp \left\{-\frac{k}{2}\left(\rho_{1}+\rho_{2}\right)\right\} \sum_{l} P_{l}\left(\cos \theta_{12}\right) F_{l}\left(\rho_{1}, \rho_{2}\right),
$$

where $P_{l}$ is the Legendre's polynomial of $l$-th order, $\theta_{12}$ is the angle between vectors $\boldsymbol{\rho}_{1}, \boldsymbol{\rho}_{2}$,

$$
F_{l}\left(\rho_{1}, \rho_{2}\right)=\sum_{m, n} A_{l m n}\left\{\rho_{1}^{m} \rho_{2}^{n}+\rho_{1}^{n} \rho_{2}^{m}\right\},
$$

$l \geqslant 0, m \geqslant n \geqslant 0, k$ and $A_{l m n}$ are the variational parameters. The function (9) with 41 parameters gives the ion energy $E_{-}=-1.0526 \mathrm{Ry}$.

It was shown from the following examples that the increase of the number of variational parameters improve description accuracy of quantum state of the $H^{-}$ion. However, with small number of parameters, the description is inaccurate, and wave functions with the large number of parameters are very cumbersome and inconvenient for following use. The aesthetic side of the case also plays a role.

\section{The basis-variational approach}

The construction of optimal wave function, which is compact and gives a fairly accurate description of the $H^{-}$ion, can be done using the reference system approach generalizing the Schull-Löwdin function [9]. As follows from the formula (3), this function is the symmetric combination of $1 s$-type one-electron functions in the neutral hydrogen atom,

$$
\begin{aligned}
\varphi_{1 s}(\rho)=\frac{1}{\sqrt{4 \pi}} R_{1 s}(\rho), & R_{1 s}(\rho)=2 \alpha_{1}^{3 / 2} e^{-\alpha_{1} \rho}, \\
\varphi_{1 s^{\prime}}(\rho)=\frac{1}{\sqrt{4 \pi}} R_{1 s^{\prime}}(\rho), & R_{1 s^{\prime}}(\rho)=2 \alpha_{2}^{3 / 2} e^{-\alpha_{2} \rho} .
\end{aligned}
$$

This basis can be extended including in it the functions which are analogues of $2 p, 3 d, 4 f$-functions of electron in the hydrogen atom, namely

$$
\begin{array}{ll}
\varphi_{2 p, m}(\boldsymbol{\rho})=Y_{1, m}(\theta, \varphi) R_{2 p}(\rho), & R_{2 p}(\rho)=\frac{2}{\sqrt{3}} \beta_{1}^{5 / 2} \rho e^{-\beta_{1} \rho} \\
\varphi_{2 p^{\prime}, m}(\boldsymbol{\rho})=Y_{1, m}(\theta, \varphi) R_{2 p^{\prime}}(\rho), & R_{2 p^{\prime}}(\rho)=\frac{2}{\sqrt{3}} \beta_{2}^{5 / 2} \rho e^{-\beta_{2} \rho} \\
\varphi_{3 d, m}(\boldsymbol{\rho})=Y_{2, m}(\theta, \varphi) R_{3 d}(\rho), & R_{3 d}(\rho)=\frac{2 \sqrt{2}}{3 \sqrt{5}} \gamma_{1}^{7 / 2} \rho^{2} e^{-\gamma_{1} \rho} \\
\varphi_{3 d^{\prime}, m}(\boldsymbol{\rho})=Y_{2, m}(\theta, \varphi) R_{3 d^{\prime}}(\rho), & R_{3 d^{\prime}}(\rho)=\frac{2 \sqrt{2}}{3 \sqrt{5}} \gamma_{2}^{7 / 2} \rho^{2} e^{-\gamma_{2} \rho}
\end{array}
$$

etc., where $Y_{l, m}(\theta, \varphi)$ are the spherical functions and $\beta_{1}, \beta_{2}, \gamma_{1}, \gamma_{2}, \ldots$ are the variational parameters. Due to the presence of factors $Y_{l, m}(\theta, \varphi)$ the functions which belong to different shells are orthogonal to each other.

Considering the addition rule of the spherical functions

$$
\sum_{m=-l}^{l} Y_{l, m}\left(\theta_{1}, \varphi_{1}\right) Y_{l,-m}\left(\theta_{2}, \varphi_{2}\right)=\frac{2 l+1}{4 \pi} P_{l}(t),
$$

where $t$ is the cosine of the angle between vectors $\boldsymbol{\rho}_{1}$ and $\boldsymbol{\rho}_{2}$, let us create set of the two-electron functions from (11), (12) 


$$
\begin{aligned}
& \psi_{1}\left(\boldsymbol{\rho}_{1}, \boldsymbol{\rho}_{2}\right)=\frac{N_{1}}{\sqrt{2}}\left\{R_{1 s}\left(\rho_{1}\right) R_{1 s^{\prime}}\left(\rho_{2}\right)+R_{1 s}\left(\rho_{2}\right) R_{1 s^{\prime}}\left(\rho_{1}\right)\right\} \frac{1}{4 \pi} P_{0}(t) ; \\
& \psi_{2}\left(\boldsymbol{\rho}_{1}, \boldsymbol{\rho}_{2}\right)=\frac{N_{2}}{\sqrt{2}}\left\{R_{2 p}\left(\rho_{1}\right) R_{2 p^{\prime}}\left(\rho_{2}\right)+R_{2 p}\left(\rho_{2}\right) R_{2 p^{\prime}}\left(\rho_{1}\right)\right\} \frac{\sqrt{3}}{4 \pi} P_{1}(t) ; \\
& \psi_{3}\left(\boldsymbol{\rho}_{1}, \boldsymbol{\rho}_{2}\right)=\frac{N_{3}}{\sqrt{2}}\left\{R_{3 d}\left(\rho_{1}\right) R_{3 d^{\prime}}\left(\rho_{2}\right)+R_{3 d}\left(\rho_{2}\right) R_{3 d^{\prime}}\left(\rho_{1}\right)\right\} \frac{\sqrt{5}}{4 \pi} P_{2}(t)
\end{aligned}
$$

etc. The functions (14) are normalized,

$$
\begin{aligned}
& N_{1}=\left\{1+\frac{\left(4 \alpha_{1} \alpha_{2}\right)^{3}}{\left(\alpha_{1}+\alpha_{2}\right)^{6}}\right\}^{-1 / 2}, \\
& N_{2}=\left\{1+\frac{\left(4 \beta_{1} \beta_{2}\right)^{5}}{\left(\beta_{1}+\beta_{2}\right)^{10}}\right\}^{-1 / 2}, \\
& N_{3}=\left\{1+\frac{\left(4 \gamma_{1} \gamma_{2}\right)^{7}}{\left(\gamma_{1}+\gamma_{2}\right)^{14}}\right\}^{-1 / 2}, \quad \ldots
\end{aligned}
$$

Due to orthogonality of Legendre polynomials the functions (14) are orthogonal to each other and their radial factors are generalized the Schull-Löwdin function. Therefore the functions $\psi_{i}\left(\boldsymbol{\rho}_{1}, \boldsymbol{\rho}_{2}\right)$ form the two-electron basis in space $\{\boldsymbol{R}\} \equiv\left\{\boldsymbol{\rho}_{1}, \boldsymbol{\rho}_{2}\right\}$, which allows us to search the equation solution (1) in the series form

$$
\Psi_{-}\left(\boldsymbol{\rho}_{1}, \boldsymbol{\rho}_{2}\right)=\sum_{s=1}^{k} a_{s} \psi_{s}\left(\boldsymbol{\rho}_{1}, \boldsymbol{\rho}_{2}\right) .
$$

Substituting the expression (16) in the equation (1), multiplying by $\psi_{s}\left(\boldsymbol{\rho}_{1}, \boldsymbol{\rho}_{2}\right)$ and integrating by coordinates we obtain the system of linear homogeneous equations for unknown coefficients $a_{s}$ :

$$
\begin{aligned}
& a_{1}\left(H_{11}-E_{-}\right)+a_{2} V_{12}+a_{3} V_{13}+\cdots+a_{k} V_{1 k}=0 ; \\
& a_{1} V_{21}+a_{2}\left(H_{22}-E_{-}\right)+a_{3} V_{23}+\cdots+a_{k} V_{2 k}=0 \text {; } \\
& a_{1} V_{k 1}+a_{2} V_{k 2}+a_{3} V_{k 3}+\cdots+a_{k}\left(H_{k k}-E_{-}\right)=0 .
\end{aligned}
$$

The normalization condition of function (16) requires satisfaction of the expression

$$
\sum_{s=1}^{k} a_{s}^{2}=1 .
$$

The matrix elements which appear in the equations (17) are defined as follows:

$$
\begin{aligned}
& H_{i i}=\left\langle\psi_{i}\left|\hat{h}\left(\mathbf{r}_{1}\right)+\hat{h}\left(\mathbf{r}_{2}\right)+\hat{v}\left(\mathbf{r}_{1}, \mathbf{r}_{2}\right)\right| \psi_{i}\right\rangle ; \\
& H_{i j} \equiv V_{i j}=\left\langle\psi_{i}\left|\hat{v}\left(\mathbf{r}_{1}, \mathbf{r}_{2}\right)\right| \psi_{j}\right\rangle \quad \text { by } \quad i \neq j .
\end{aligned}
$$

All of them are calculated in the analytical form. In particular,

$$
\begin{aligned}
& \left\langle\psi_{1}\left|\hat{h}\left(\mathbf{r}_{1}\right)+\hat{h}\left(\mathbf{r}_{2}\right)\right| \psi_{1}\right\rangle=N_{1}^{2}\left\{\alpha_{1}^{2}+\alpha_{2}^{2}-2\left(\alpha_{1}+\alpha_{2}\right)+2 \frac{\left(4 \alpha_{1} \alpha_{2}\right)^{3}}{\left(\alpha_{1}+\alpha_{2}\right)^{6}}\left[\alpha_{1} \alpha_{2}-\alpha_{1}-\alpha_{2}\right]\right\} \mathrm{Ry} ; \\
& \left\langle\psi_{2}\left|\hat{h}\left(\mathbf{r}_{1}\right)+\hat{h}\left(\mathbf{r}_{2}\right)\right| \psi_{2}\right\rangle=N_{2}^{2}\left\{\beta_{1}^{2}+\beta_{2}^{2}-\beta_{1}-\beta_{2}+\frac{\left(4 \beta_{1} \beta_{2}\right)^{5}}{\left(\beta_{1}+\beta_{2}\right)^{10}}\left[2 \beta_{1} \beta_{2}-\beta_{1}-\beta_{2}\right]\right\} \mathrm{Ry} ; \\
& \left\langle\psi_{3}\left|\hat{h}\left(\mathbf{r}_{1}\right)+\hat{h}\left(\mathbf{r}_{2}\right)\right| \psi_{3}\right\rangle=N_{3}^{2}\left\{\gamma_{1}^{2}+\gamma_{2}^{2}-\frac{2}{3}\left(\gamma_{1}+\gamma_{2}\right)+\frac{\left(4 \gamma_{1} \gamma_{2}\right)^{7}}{\left(\gamma_{1}+\gamma_{2}\right)^{14}}\left[2 \gamma_{1} \gamma_{2}-\frac{2}{3}\left(\gamma_{1}+\gamma_{2}\right)\right]\right\} \mathrm{Ry}
\end{aligned}
$$


etc. Using the expansion of factor $\left|\boldsymbol{\rho}_{1}-\boldsymbol{\rho}_{2}\right|^{-1}$ in a series of Legendre polynomials $P_{l}(t)$ we reduce the calculation of matrix elements of inter-electron interactions operator to the integrals calculation of type

$$
I_{n \mid m}(a \mid b)=\int_{0}^{\infty} d \rho_{1} \rho_{1}^{n} e^{-a \rho_{1}} \int_{0}^{\rho_{1}} d \rho_{2} \rho_{2}^{m} e^{-b \rho_{2}} .
$$

The explicit expressions of the matrix elements $V_{i j}$ through the integrals (21) are given in the Appendix. The value of energy of $H^{-}$ion have found by minimizing the root of the secular equation

$$
\operatorname{det}\left|H_{i j}-E_{-} \delta_{i j}\right|=0
$$

with regard to parameters $\alpha_{1}, \alpha_{2}, \ldots, \gamma_{2}$. It turned out that the minimum energy value is achieved by $\beta_{1}=\beta_{2}, \gamma_{1}=\gamma_{2}, \delta_{1}=\delta_{2}, \ldots$. In order to find the coefficients of expansion $a_{s}$ let us use $k-1$ equation of system (17) and the normalization condition (18) at the known value of $E_{-}$. The calculation results of the ion energy, variational parameters and coefficients of expansion $a_{s}$ for different values of number of terms in expansion $(1 \leqslant k \leqslant 4)$ are given in Table 1.

Table 1. The value of $H^{-}$ion energy, variational parameters and the coefficients of expansion $a_{s}$.

\begin{tabular}{|c|c|c|c|c|}
\hline$k$ & 1 & 2 & 3 & 4 \\
\hline$E_{-}(k), \mathrm{Ry}$ & -1.0266 & -1.04915 & -1.05058 & -1.05087 \\
\hline$\alpha_{1}$ & 1.0392 & 1.035582 & 1.03524 & 1.03518 \\
\hline$\alpha_{2}$ & 0.2832 & 0.323936 & 0.326516 & 0.32706 \\
\hline$\beta_{1}=\beta_{2}$ & - & 0.998302 & 1.00138 & 1.00151 \\
\hline$\gamma_{1}=\gamma_{2}$ & - & - & 1.53401 & 1.53982 \\
\hline$\delta_{1}=\delta_{2}$ & - & - & - & 2.09053 \\
\hline$a_{1}$ & 1.0000 & 0.993734 & 0.9939285 & 0.993977 \\
\hline$a_{2}$ & - & -0.111768 & -0.1086079 & -0.108094 \\
\hline$a_{3}$ & - & - & -0.0176175 & -0.0171053 \\
\hline$a_{4}$ & - & - & - & -0.005792 \\
\hline$c(k)$ & - & - & 0.039840 & 0.039592 \\
\hline$E_{-}(c)$ & - & - & -1.055054 & -1.0552888 \\
\hline
\end{tabular}

\section{The perturbation theory}

As can be seen from the Table 1 the convergence of series (16) become worse at $k \geqslant 3$, which require the increase of terms number of series. To omit the cumbersome function, we represent the solution of the equation (1) as the sum of two terms

$$
\Psi_{-}\left(\boldsymbol{\rho}_{1}, \boldsymbol{\rho}_{2}\right)=\Psi_{0}\left(\boldsymbol{\rho}_{1}, \boldsymbol{\rho}_{2}\right)+c \Phi\left(\boldsymbol{\rho}_{1}, \boldsymbol{\rho}_{2}\right) .
$$

Here $\Psi_{0}\left(\boldsymbol{\rho}_{1}, \boldsymbol{\rho}_{2}\right)$ is the normalized function (16) at $k=3$ or 4 , which corresponds to the energy $E_{-}(k)$ found with the known parameters $\alpha_{1}, \alpha_{2}, \beta_{1}, \beta_{2}, \gamma_{1}, \gamma_{2}, \ldots$ as well as known coefficients $a_{s}$. The factor $c$ is the variational parameter and function $\Phi\left(\boldsymbol{\rho}_{1}, \boldsymbol{\rho}_{2}\right)$ is the correction to the Schull-Löwdin function, namely

$$
\Phi\left(\rho_{1}, \rho_{2}\right)=\frac{M}{4 \pi} \alpha^{3}\left\{\left\{e^{-\alpha \rho_{1}} e^{-\frac{\alpha}{2} \rho_{2}}\left(1-\Gamma \rho_{2}\right)+e^{-\alpha \rho_{2}} e^{-\frac{\alpha}{2} \rho_{1}}\left(1-\Gamma \rho_{1}\right)\right\} .\right.
$$

The factor $M$ determines from the normalization condition $\Phi\left(\rho_{1}, \rho_{2}\right)$ and depends on the variational parameters $\alpha$ and $\Gamma$ :

$$
M=\left\{1-6 \frac{\Gamma}{\alpha}\left(1-2 \frac{\Gamma}{\alpha}\right)+\left(\frac{8}{9}\right)^{3}\left[1-4 \frac{\Gamma}{\alpha}\left(1-\frac{\Gamma}{\alpha}\right)\right]\right\}^{-1 / 2}
$$

Mathematical Modeling and Computing, Vol. 6, No. 1, pp. 144-151 (2019) 
Now we have the problem with three variational parameters, and the energy of ion can be found from the expression

$$
E_{-}(c)=\min \frac{E_{-}(k)+2 c\left\langle\Phi|\hat{H}| \Psi_{0}\right\rangle+c^{2}\langle\Phi|\hat{H}| \Phi\rangle}{1+2 c\left\langle\Phi \mid \Psi_{0}\right\rangle+c^{2}} .
$$

In our case the parameter $c$ should be several times smaller than in the approximation (4) because zero-order approximation of the function $\phi_{0}\left(\boldsymbol{\rho}_{1}, \boldsymbol{\rho}_{2}\right)$ gives more than $90 \%$ of ionization energy. On the other hand, the coefficients of the expansion (16) have such order: $a_{1} \approx 1.0 ;\left|a_{2}\right| \sim 0.1 ;\left|a_{3}\right| \sim 0.01$. Therefore, while calculating matrix elements, which appear in the expression (24), we consider the approximation

$$
\left\langle\Phi \mid \Psi_{0}\right\rangle \cong a_{1}\left\langle\Phi \mid \psi_{1}\right\rangle, \quad\left\langle\Phi|\hat{H}| \Psi_{0}\right\rangle \cong a_{1}\left\langle\Phi|\hat{H}| \psi_{1}\right\rangle .
$$

As follows from orthonormality of the functions $\psi_{i}\left(\boldsymbol{\rho}_{1}, \boldsymbol{\rho}_{2}\right)$

$$
\left\langle\Phi\left|\hat{v}\left(\mathbf{r}_{1}, \mathbf{r}_{2}\right)\right| \psi_{1}\right\rangle=2 \mathrm{Ry} .
$$

Herewith instead the matrix element $\left\langle\Phi\left|\hat{v}\left(\mathbf{r}_{1}, \mathbf{r}_{2}\right)\right| \Psi_{1}\right\rangle$ we used the corrected matrix element

$$
A\left\langle\Psi_{1}\left|\hat{v}\left(\mathbf{r}_{1}, \mathbf{r}_{2}\right)\right| \Psi_{1}\right\rangle\left\langle\Phi \mid \psi_{1}\right\rangle+(1-A)\left\langle\Phi\left|\hat{v}\left(\mathbf{r}_{1}, \mathbf{r}_{2}\right)\right| \Psi_{1}\right\rangle
$$

which effectively takes into account the influence upper terms of expansion (16). The factor $A=$ $0.5(\sqrt{3}-1)$ corresponds to the "golden section" approximation. At $k=4$ and $\Gamma=0.1001, \alpha=0.4095$ the energy minimum is achieved at $c_{0}=-0.1202$. The value of ion energy equals $E_{-}=-1.055502 \mathrm{Ry}$, that corresponds to the result of the work [7].

The normalized function (23) is presented in the form

$$
\Psi_{-}\left(\boldsymbol{\rho}_{1}, \boldsymbol{\rho}_{2}\right)=\sum_{s=1}^{4} \tilde{a}_{s} \psi_{i}\left(\boldsymbol{\rho}_{1}, \boldsymbol{\rho}_{2}\right)+\tilde{c}_{0} \Phi\left(\rho_{1}, \rho_{2}\right),
$$

where

$$
\begin{aligned}
& \tilde{a}_{s}=a_{s} \eta^{-1 / 2} \\
& \tilde{c}_{0}(k)=c_{0} \eta^{-1 / 2} \\
& \eta=1+2 c_{0} a_{1}\left\langle\Phi \mid \psi_{1}\right\rangle+c_{0}^{2}=0.910821
\end{aligned}
$$

\section{Conclusions}

As follows from the calculation of the energy, the distribution of electron density in $H^{-}$ion can be interpreted within shell model. Two of the shells correspond to the spherically-symmetric distribution of charge (the function $\psi_{1}\left(\boldsymbol{\rho}_{1}, \boldsymbol{\rho}_{2}\right)$ ), and the maximum of distribution is in the distances approximately $a_{0}$ and $3 a_{0}$ from the proton. Three other shells correspond to the distribution of charge by type $2 p-$, $3 d$ - and $4 f$-hydrogen function, and the maximum of radial distribution is in the distances $\approx 2 a_{0}$. The most significant contributions to the ionization energy gives the spherically-symmetric shells and shell of the $2 p$-type. The shell of $3 d$-type gives much lower contribution, and the contribution of $4 f$-shell is negligible. The function $\Psi_{0}\left(\boldsymbol{\rho}_{1}, \boldsymbol{\rho}_{2}\right)$ gives more than $90 \%$ of ionization energy. The correction function $\Phi\left(\boldsymbol{\rho}_{1}, \boldsymbol{\rho}_{2}\right)$ gives the contribution to the ionization energy which is approximately 5 times less than the contributions of functions $\psi_{1}\left(\boldsymbol{\rho}_{1}, \boldsymbol{\rho}_{2}\right)$ or $\psi_{2}\left(\boldsymbol{\rho}_{1}, \boldsymbol{\rho}_{2}\right)$. Nevertheless, this contribution is also important, it takes into account the radial and angular correlations between electrons from the first and third shells and allows us to achieve spectroscopic accuracy $\left(\sim 10^{-5} \mathrm{Ry}\right)$ in the calculation of the ionization energy of negative ion of hydrogen. 


\section{Appendix}

Let us show here the expressions of matrix elements of operator of inter-electron interactions $\hat{v}\left(\mathbf{r}_{1}, \mathbf{r}_{2}\right)$ which appear in the expression (19):

$$
\begin{aligned}
V_{11}= & 8 N_{1}^{2} \frac{\alpha_{1} \alpha_{2}}{\left(\alpha_{1}+\alpha_{2}\right)^{4}}\left\{\alpha_{1} \alpha_{2}\left(\alpha_{1}+\alpha_{2}\right)+\frac{\alpha_{1}^{3}+\alpha_{2}^{3}}{4}+5 \frac{\left(\alpha_{1} \alpha_{2}\right)^{2}}{\alpha_{1}+\alpha_{2}}\right\} \\
V_{22}= & \frac{64}{9} N_{2}^{2}\left(\beta_{1} \beta_{2}\right)^{5}\left\{I_{3 \mid 4}^{\mathrm{s}}\left(2 \beta_{1} \mid 2 \beta_{2}\right)+I_{3 \mid 4}\left(\beta_{1}+\beta_{2} \mid \beta_{1}+\beta_{2}\right)\right. \\
& \left.+\frac{2}{5}\left[I_{1 \mid 6}^{\mathrm{s}}\left(2 \beta_{1} \mid 2 \beta_{2}\right)+I_{1 \mid 6}\left(\beta_{1}+\beta_{2} \mid \beta_{1}+\beta_{2}\right)\right]\right\} ; \\
V_{33}= & N_{3}^{2} \frac{1}{25}\left(\frac{4}{3}\right)^{4}\left(\gamma_{1} \gamma_{2}\right)^{7}\left\{I_{5 \mid 6}^{\mathrm{s}}\left(2 \gamma_{1} \mid 2 \gamma_{2}\right)+I_{5 \mid 6}\left(\gamma_{1}+\gamma_{2} \mid \gamma_{1}+\gamma_{2}\right)\right. \\
& +\frac{2}{7}\left[I_{3 \mid 8}^{\mathrm{s}}\left(2 \gamma_{1} 2 \gamma_{2}\right)+I_{3 \mid 8}\left(\gamma_{1}+\gamma_{2} \mid \gamma_{1}+\gamma_{2}\right)+I_{3 \mid 8}\left(\gamma_{1}+\gamma_{2} \mid \gamma_{1}+\gamma_{2}\right)\right. \\
& \left.\left.+I_{1 \mid 10}^{\mathrm{s}}\left(2 \gamma_{1} 2 \gamma_{2}\right)+I_{1 \mid 10}\left(\gamma_{1}+\gamma_{2} \mid \gamma_{1}+\gamma_{2}\right)\right]\right\} \\
V_{12}= & N_{1} N_{2} \frac{64}{3 \sqrt{3}}\left(\alpha_{1} \alpha_{2}\right)^{3 / 2}\left(\beta_{1} \beta_{2}\right)^{5 / 2}\left\{I_{1 \mid 4}^{\mathrm{s}}\left(\alpha_{1}+\beta_{1} \mid \alpha_{2}+\beta_{2}\right)+I_{1 \mid 4}^{\mathrm{s}}\left(\alpha_{2}+\beta_{1} \mid \alpha_{1}+\beta_{2}\right)\right\} ; \\
V_{13}= & N_{1} N_{3} \frac{128}{45 \sqrt{5}}\left(\alpha_{1} \alpha_{2}\right)^{3 / 2}\left(\gamma_{1} \gamma_{2}\right)^{7 / 2}\left\{I_{1 \mid 6}^{\mathrm{s}}\left(\alpha_{1}+\gamma_{1} \mid \alpha_{2}+\gamma_{2}\right)+I_{1 \mid 6}^{\mathrm{s}}\left(\alpha_{1}+\gamma_{2} \mid \alpha_{2}+\gamma_{1}\right)\right\} ; \\
V_{23}= & N_{2} N_{3} \frac{256}{9 \cdot 15 \sqrt{15}}\left(\beta_{1} \beta_{2}\right)^{5 / 2}\left(\gamma_{1} \gamma_{2}\right)^{7 / 2}+\left\{I_{3 \mid 6}^{\mathrm{s}}\left(\beta_{1}+\gamma_{1} \mid \beta_{2}+\gamma_{2}\right)\right. \\
& \left.+I_{3 \mid 6}^{\mathrm{s}}\left(\beta_{1}+\gamma_{2} \mid \beta_{2}+\gamma_{1}\right)+\frac{9}{14}\left[I_{1 \mid 8}^{\mathrm{s}}\left(\beta_{1}+\gamma_{1} \mid \beta_{2}+\gamma_{2}\right)+I_{1 \mid 8}^{\mathrm{s}}\left(\beta_{1}+\gamma_{2} \mid \beta_{2}+\gamma_{1}\right)\right]\right\} ;
\end{aligned}
$$

etc. All matrix elements are dimensionless and expressed in Ry. Here are also introduced the symmetric integrals (21), namely

$$
I_{n \mid m}^{\mathrm{s}}(a \mid b) \equiv \frac{1}{2}\left\{I_{n \mid m}(a \mid b)+I_{n \mid m}(b \mid a)\right\} .
$$

The matrix elements and overlap integrals which appear in (24), (26) are also calculated in analytical form or reduced to the tabular integrals:

$$
\begin{aligned}
\left\langle\Phi \mid \psi_{1}\right\rangle=16 & \sqrt{2} M N_{1} \alpha^{3}\left(\alpha_{1} \alpha_{2}\right)^{3 / 2}\left\{\frac{\alpha_{2}+\frac{\alpha}{2}-3 \Gamma}{\left(\alpha+\alpha_{1}\right)^{3}\left(\alpha_{2}+\frac{\alpha}{2}\right)^{4}}+\frac{\alpha_{1}+\frac{\alpha}{2}-3 \Gamma}{\left(\alpha+\alpha_{2}\right)^{3}\left(\alpha_{1}+\frac{\alpha}{2}\right)^{4}}\right\} ; \\
\left\langle\Phi\left|\hat{v}_{12}\right| \psi_{1}\right\rangle= & \frac{16}{\sqrt{2}} M N_{1} \alpha^{3}\left(\alpha_{1} \alpha_{2}\right)^{3 / 2}\left\{I_{12}\left(\alpha+\alpha_{1}, \alpha_{2}+\frac{\alpha}{2}\right)-\Gamma I_{13}\left(\alpha+\alpha_{1}, \alpha_{2}+\frac{\alpha}{2}\right)\right. \\
+ & I_{12}\left(\alpha_{1}+\frac{\alpha}{2}, \alpha+\alpha_{2}\right)-\Gamma I_{22}\left(\alpha_{1}+\frac{\alpha}{2}, \alpha+\alpha_{2}\right)+I_{12}\left(\alpha+\alpha_{2}, \alpha_{1}+\frac{\alpha}{2}\right) \\
- & \left.\Gamma I_{13}\left(\alpha+\alpha_{2}, \alpha_{1}+\frac{\alpha}{2}\right)+I_{12}\left(\alpha_{2}+\frac{\alpha}{2}, \alpha+\alpha_{1}\right)-\Gamma I_{22}\left(\alpha_{2}+\frac{\alpha}{2}, \alpha+\alpha_{1}\right)\right\} ; \\
\left\langle\Phi\left|\hat{h}_{1}+\hat{h}_{2}\right| \psi_{1}\right\rangle= & \frac{32}{\sqrt{2}} M N_{1} \alpha^{3}\left(\alpha_{1} \alpha_{2}\right)^{3 / 2}\left\{\frac{\alpha_{1}(\alpha-1)+\alpha_{2}\left(\frac{\alpha}{2}-1\right)-\frac{3}{2} \alpha}{\left(\alpha+\alpha_{1}\right)^{3}\left(\alpha_{2}+\frac{\alpha}{2}\right)^{3}}+\frac{\alpha_{2}(\alpha-1)+\alpha_{1}\left(\frac{\alpha}{2}-1\right)-\frac{3}{2} \alpha}{\left(\alpha+\alpha_{2}\right)^{3}\left(\alpha_{1}+\frac{\alpha}{2}\right)^{3}}\right. \\
& -\frac{\Gamma\left[\alpha_{2}\left(\alpha-\alpha_{2}\right)-2 \alpha_{2}+3 \alpha_{1}(\alpha-1)-4 \alpha\right]}{\left(\alpha+\alpha_{1}\right)^{3}\left(\alpha_{2}+\frac{\alpha}{2}\right)^{4}} \\
& \left.-\frac{\Gamma\left[\alpha_{1}\left(\alpha-\alpha_{1}\right)-2 \alpha_{1}+3 \alpha_{2}(\alpha-1)-4 \alpha\right]}{\left(\alpha+\alpha_{2}\right)^{3}\left(\alpha_{1}+\frac{\alpha}{2}\right)^{4}}\right\} .
\end{aligned}
$$


[1] Wildt R. The Continuous Spectrum of Stellar Atmospheres Consisting Only of Atoms and Negative Ions of Hydrogen. Astrophys. Journ. 93, 47-51 (1941).

[2] Chandrasekhar S., Breen F. H. On the Continuous Absorption Coefficient of the Negative Hydrogen Ion. III. Astrophys. Journ. 104, 430-445 (1946).

[3] Geltman S. The Bound-Free Absorption Coefficient of the Hydrogen Negative Ion. Astrophys. Journ. 136, 935-945 (1962).

[4] Wishart A. W. The Bound-Free Photodetachment Cross Section of $H^{-}$. J. Phys. B: Atom. Molec. Phys. 12 (21), 3511-3519, (1979).

[5] Vavrukh M. V., Vasil'eva I. E., Stelmakh O. M., Tyshko N. L. Continuous Absorption and Depression in the Solar Spectrum at Wavelengths from 650 to $820 \mathrm{~nm}$. Kinematics and Physics of Celestial Bodies. 32 (3), 129-144 (2016).

[6] Hylleraas E. A. Die Elektronenaffinität des Wasserstoffatoms nach der Wellenmechanik. Zeitschrift für Physik. 60, 624-630 (1930).

[7] Pekeris C. L. $1^{1} S, 2^{1} S$ and $2^{3} S$ States of $H^{-}$and of He. Phys. Rev. 126, 1470-1476 (1962).

[8] Massey H. S. W. Negative Ions. Cambridge University Press (1976).

[9] Schull H., Löwdin P.-O. Correlation Splitting in Helium-Like Ions. J. Chem. Phys. 25, 1035-1040 (1956).

[10] Kinoshita T. Ground State of the Helium Atom. Phys. Rev. 105, 1490-1502 (1957).

[11] Hart J. F., Herzberg G. Twenty-Parameter Eigenfunctions and Energy Values of the Ground States of He and He-Like Ions. Phys. Rev. 106, 79-82 (1957).

[12] Tweed R. J. Correlated wavefunctions for helium-like atomic systems. J. Phys. B: Atom. Molec. Phys. 5, 810-819 (1972).

\title{
Оболонкова модель електронної структури негативного іона водню
}

\author{
Ваврух М., Дзіковський Д., Стельмах О. \\ Львівсъкий національний університет імені Івана Франка, \\ вул. Кирила і Мефодія, 8, Львів, 79005, Україна
}

\begin{abstract}
У межах нерелятивістського наближення одержано компактний наближений розв'язок рівняння Шредингера для іона $H^{-}$у вигляді розкладу за поліномами Лежандра і варіаційними функціями типу Шулля-Льовдіна. Точність розрахунку енергії іона відповідає результатам, одержаним за допомогою багатопараметричних функцій Гіллераса і Пекеріса.

Ключові слова: негативний іон водню, базисний підхід, варіаційний підхід, енергія іонізації.
\end{abstract}

2000 MSC: $70 \mathrm{~F} 05$

Удк: 523.9, 523.9-7, 523.9-47 